Musées, Patrimoine et Culture scientifiques et techniques

$117 \mid 2008$

mai - juin 2008

\title{
Des dispositifs interactifs sur l'Espace dans les musées
}

Gérard Azoulay

\section{OpenEdition \\ Journals}

Édition électronique

URL : http://journals.openedition.org/ocim/330

DOI : 10.4000/ocim.330

ISSN : 2108-646X

Éditeur

OCIM

Édition imprimée

Date de publication : 1 mai 2008

Pagination : 27-33

ISSN : 0994-1908

Référence électronique

Gérard Azoulay, «Des dispositifs interactifs sur l'Espace dans les musées », La Lettre de l'OCIM [En ligne], 117| 2008, mis en ligne le 01 décembre 2010, consulté le 01 mai 2019. URL : http:// journals.openedition.org/ocim/330 ; DOI : 10.4000/ocim.330 
Il faut cependant préciser qu'à l'exception de quelques rares éléments patrimoniaux, les activités spatiales laissent très peu de traces sur Terre pour alimenter ces réflexions. Ce n'est pas faute d'avoir, depuis toujours, exercé sur l'homme une attirance qui s'est traduite par une profusion d'œuvres fictionnelles : le premier voyage dans l'Espace ne remonte-t-il pas à Lucien de Samosate au II ${ }^{\text {e }}$ siècle ? Cette absence de traces concrètes rend l'Espace difficile à appréhender. Les images qui nous parviennent des dernières missions spatiales, superbes et surprenantes, s'en chargent en partie ; la publicité ou le cinéma qui s'emparent de l'iconologie de l'Espace, certaines de façon étonnamment triviale, y contribuent également. Mais afin de dépasser la fascination directe exercée par ces productions visuelles, et conscient du pouvoir d'attraction intrinsèque de l'Espace, il nous semblait essentiel de nous interroger sur les moyens de faire percevoir la complexité de ce milieu, et sur les dispositifs susceptibles de traduire pour un public néophyte la richesse du nouveau monde qui s'offre à lui du point de vue des connaissances, des représentations, ou encore des sensations.

Une des difficultés majeures à surmonter pour rendre tangibles les apports de l'Espace et en faciliter la compréhension réside dans le fait qu'une grande partie des mesures et des expériences réalisées à partir des instruments spatiaux ne sont pas accessibles aisément au public, ni même aux médiateurs. En soi ces informations ne sont pas cachées, mais elles sont complexes et longues à être rassemblées ; elles requièrent des processus délicats et coûteux de transformation pour être visualisables. Enfin, la synthèse et la visualisation des données spatiales nécessitent d'élaborer de nouveaux processus de représentation ou d'expérimentation qui permettent de transcrire la richesse des apports spatiaux.

Tout l'enjeu de notre approche a été de rechercher des traductions du milieu spatial et de ses caractéristiques, par des techniques qui ne se rapportent pas seulement à l'audiovisuel ou au texte, ce qui nous aurait alors renvoyé au registre du récit, de la narration mais qui relèvent plutôt de l'ordre de l'expérience et de la manipulation directe des mesures. Notre intention était d'imaginer des dispositifs interactifs qui proposeraient au visiteur un autre rapport au monde : celui que peut nous offrir aujourd'hui le regard porté depuis l'Espace.

Ces contraintes ont conduit à concevoir une famille de dispositifs interactifs bilingues (français et anglais) qui fournissent des informations en temps réel informatique, régulièrement mises à jour. Ils sont pour l'instant au nombre de quatre, et chacun d'entre eux a nécessité la création de logiciels d'autant plus sophistiqués que l'appareil devait être aussi simple à utiliser que l'un de ces jeux électroniques pour le grand public qui sont immédiatement accessibles à ceux qui les manipulent.

Pour mettre à la portée de tous cette richesse culturelle, l'Observatoire de l'Espace du CNES (Centre National d'Études Spatiales) ne dispose, ni ne souhaite disposer, de lieux assez nombreux pour toucher un large public. Aussi, pour la diffusion d'un tel projet, ses interlocuteurs naturels sont les musées et les établissements culturels avec lesquels il noue des partenariats dans des conditions très souples de collaboration.

\section{Des expériences irremplaçables}

La logique qui a prévalu au développement de ces dispositifs était de répondre aux questions que pose l'accès à l'espace depuis la deuxième moitié du XXe siècle, celle de l'expérience de la micropesanteur avec le Stationaute, celle de l'accroissement des savoirs avec la Spatiosphère, et enfin la question de la représentation de notre monde avec l'Hydronaute et l'Orbiscope.

\section{Un musée en orbite terrestre}

Cet outil, le dernier-né des dispositifs interactifs, révèle une vision de la Terre et de son environnement totalement inédite. L'utilisateur a la possibilité de visualiser combien la Terre, depuis le début de l'ère spatiale, est entourée d'une énorme quantité d'objets en orbite qu'ils soient ou non en fonctionnement. Cette application informatique exploite les observations faites par des radars terrestres qui suivent en permanence ces objets, à partir d'une taille supérieure à dix centimètres. Grâce à un écran tactile et un joystick, l'utilisateur choisit son mode de consultation, et peut accéder à une grande variété d'images qui sont étayées par des informations précises.

La Terre s'offre à lui, bleue sur fond noir, mais littéralement cernée de points brillants, qui l'entourent, et lui font une auréole. Nous savons tous que des satellites tournent autour d'elle. Nous observons parfois le passage de la Station spatiale internationale par les nuits claires. Mais constater à quel point ils sont nombreux est vraiment une surprise, d'autant plus que tous les objets - satellites ou débris - nous sont restitués sur un écran. Nous pouvons 


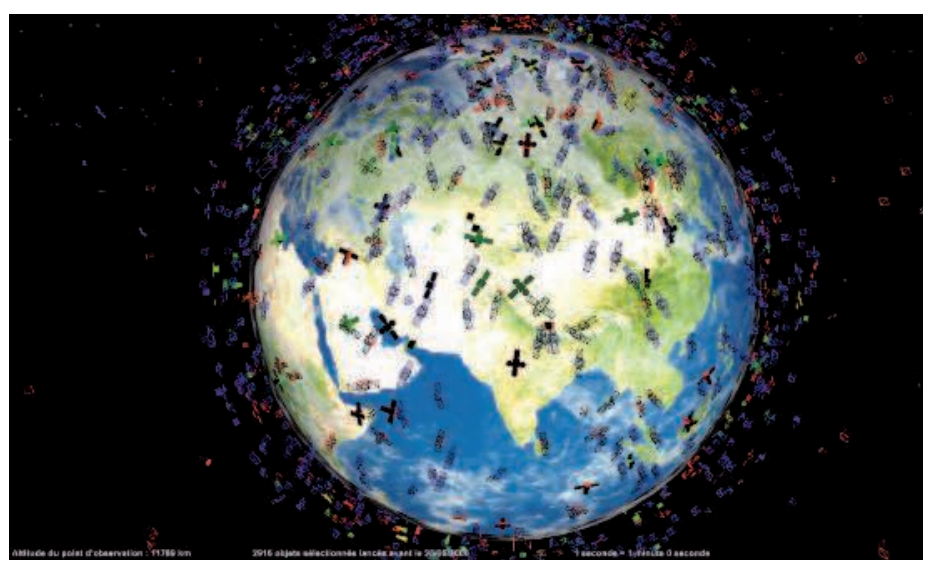

L'écran de visualisation de l'Orbiscope : le mode global avec satellites en fonctionnement et hors service ๑) CNES

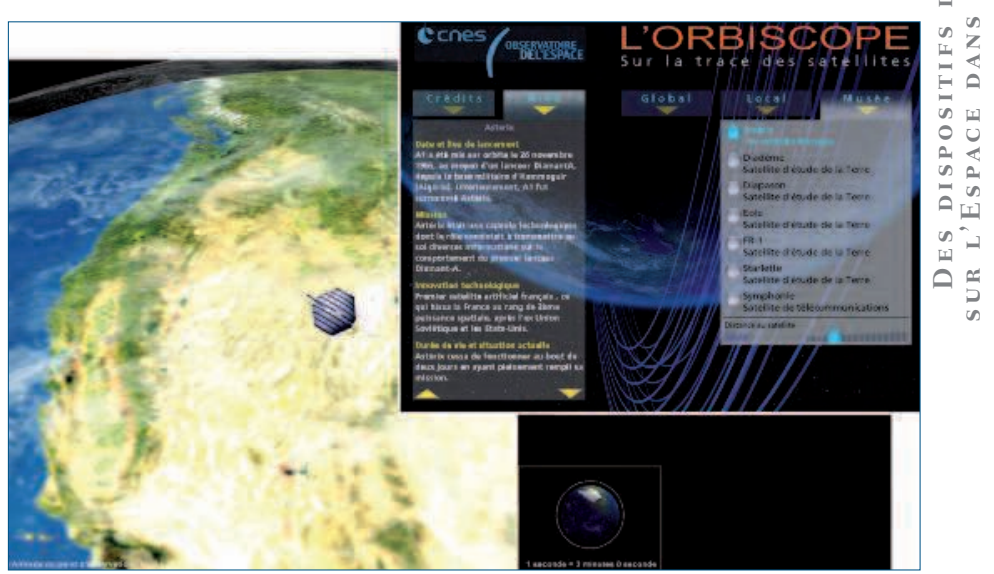

L'écran de visualisation de l'Orbiscope le mode « musée » avec le satellite Asterix (c) CNES nous en approcher ou nous en éloigner afin d'en embrasser la totalité. Nous visitons l'Espace, nous l'utilisons pour nous observer nous-mêmes, ce qui est désormais indispensable, et en même temps nous le peuplons de divers débris qui tourneront en orbite durant de très longues périodes. L'Orbiscope offre à la fois une prise de conscience de ce que l'exploration de l'Espace représente pour les hommes mais aussi des résidus que l'aventure spatiale a engendrés, avec les objets laissés en orbite.

L'utilisateur de l'Orbiscope a trois modes possibles de consultation, et chacun est assorti d'une multitude de précisions accessibles.

Le mode global lui permet de choisir l'époque où il souhaite se situer. Les objets en orbite sont de plus en plus nombreux au fur et à mesure qu'on s'éloigne des origines de l'aventure spatiale, qui n'est vieille pourtant que d'une cinquantaine d'années. L'utilisateur mesure la rapidité de notre intrusion dans l'Espace. Il reconnait le type des objets, distingue les satellites : astronomie, télécommunications, observation de la Terre, sait s'ils sont ou non en activité, voit les débris laissés par cette aventure. Il identifie le pays d'origine des satellites, ainsi que le type d'orbite où ils se situent - géostationnaire, polaire, ou de transfert. Tous ces critères lui donnent une perception globale de l'environnement de notre planète.

Le mode local lui permet de s'associer à un satellite en activité. Il parcourt avec lui son orbite, et peut visualiser les données spécifiques qu'il perçoit. Il reçoit les données et les images que le satellite qu'il a choisi enregistre. Il voit les océans avec l'œil du satellite océanographique Jason. Il regarde évoluer la végétation comme la perçoit SPOT 5. Il prend connaissance de ce qu'est l'atmosphère vue par
Météosat. Il découvre les paysages terrestres depuis la Station spatiale internationale. Ce parcours avec les satellites permet d'accéder à un champ d'expérience extrêmement varié.

Le mode « musée » propose de voir quelques satellites historiques français, donne des informations à leur sujet, les replace dans leur contexte scientifique et politique. Cela permet de tracer une expérience, une histoire de l'Espace spécifiquement française et souvent méconnue.

Ces trois modes de consultation se complètent et permettent d'appréhender des informations très détaillées sur un milieu où les avancées techniques et scientifiques ne laissent pas de traces tangibles.

\section{Des nouvelles représentations de l'Océan}

Pour des générations d'hommes, l'Océan, qui recouvre $70 \%$ de la surface de la Terre, est demeuré un milieu difficile d'accès, avec ses zones d'ombres et ses mécanismes difficilement compréhensibles. Les pêcheurs y voyaient un outil de travail, les aventuriers et les découvreurs, une chance d'enrichissement personnel et de gloire politique, les plaisanciers, un terrain d'amusement. Mais aucun n'était pleinement conscient de ce que l'Océan recelait dans ses profondeurs, ni du rôle de ses courants dans l'équilibre climatique de la planète Terre, et dans la vie de ses habitants.

Les médias nous parlent constamment de l'influence des Océans sur les changements climatiques. Cela suscite l'envie d'aller voir plus loin, de s'informer, de comprendre le comment et le pourquoi. Désormais, l'Hydronaute permet, grâce à l'usage des mesures satellitaires (les satellites francoaméricains Topex-Poséidon et Jason, l'instrument franco-japonais Polder, le satellite européen ERS1) un accès facile à ce qui reste un milieu complexe. 


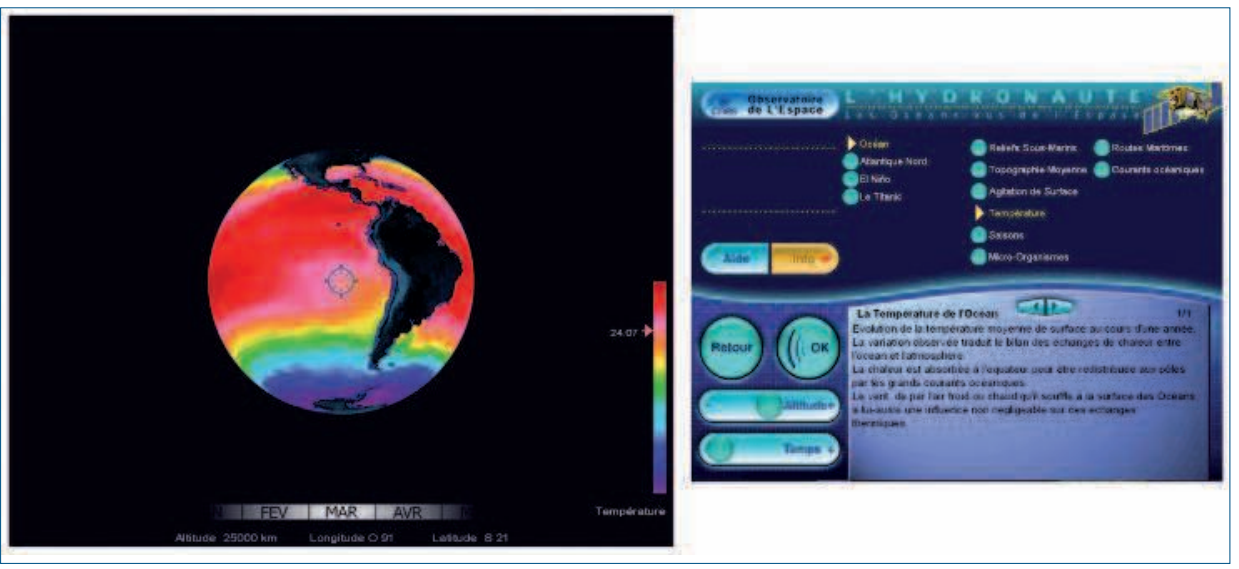

Les écrans de contrôle et de visualisation de l'Hydronaute : la température moyenne de l'Océan (c) CNES

L'Hydronaute est un dispositif avec lequel on navigue grâce à une interface conviviale et ergonomique : un joystick et un écran tactile. Il permet à chacun de prendre conscience des nombreuses interactions dont l'Océan est le siège inattendu et de notre responsabilité d'hommes. Nous pouvons influer, entre autres, sur les changements climatiques ou l'appauvrissement des ressources halieutiques, dont dépend la survie de l'humanité. Cet outil rend visible les principales découvertes qui ont été faites avec les satellites.

Les mers ne sont pas plates, contrairement aux apparences. Leur surface se bombe au-dessus d'un volcan sous-marin, et se creuse au-dessus des grandes fosses. Les mesures altimétriques montrent très précisément que loin d'être un long fleuve tranquille, chaque courant est agité de méandres et de tourbillons. Les données satellitaires affinent en permanence la description des structures océaniques. La surface océanique est une mine d'informations sur les échanges thermiques entre l'Océan et l'atmosphère qui stabilisent la température de la Terre. Les grands courants océaniques génèrent les circulations cycloniques et anticycloniques dont l'impact sur les variations climatiques est majeur ; ils évoluent dans le temps. La température de l'Océan varie selon la latitude : les grands courants océaniques et les vents redistribuent les différences de température de la surface de la Terre, qui reçoit plus d'énergie à l'équateur qu'aux pôles.

Dans le domaine du vivant et de l'Océan biologique, par exemple, en offrant la possibilité de visualiser l'évolution du phytoplancton, sur une échelle temporelle, l'Hydronaute offre une information essentielle pour comprendre les écosystèmes marins. Il donne aussi la possibilité de suivre les différentes espèces migratrices : tortues, baleine ou encore albatros afin d'en mieux saisir la nature des parcours. Un mode local permet ensuite au visiteur de s'immerger dans des bassins tridimensionnels et d'observer le déplacement de la bosse d'eau chaude due au phénomène $E l$ niño, de jeter des bouées dans les courants de l'Atlantique Nord afin de saisir de quelle manière des marées noires peuvent envahir nos côtes ou encore d'observer l'effet du Mistral, un vent extrêmement puissant qui descend le couloir rhodanien, sur la température de surface de la Méditerranée.

\section{L'Espace et les champs du savoir}

La Spatiosphère propose de dévoiler aux visiteurs des musées ce que l'Espace apporte à leur vie quotidienne, à leur imaginaire et aux différents champs du savoir. Par l'usage d'une commande unique - une boule tactile - l'utilisateur de la Spatiosphère peut aborder directement le sujet qui l'intéresse. Sur l'écran de visualisation, une sphère tourne de manière synchrone à la boule de commande et offre au visiteur un choix de questions - du rôle de la gravité dans le fonctionnement du cerveau à l'influence de l'espace sur les jouets - dont la réponse est fournie au travers d'un court-métrage. Un mode de recherche approfondie, davantage destiné au visiteur averti, est également disponible pour trouver un film en fonction des mots clés utilisés par ceux qui sont familiers de l'univers spatial. La Spatiosphère représente une base de données audiovisuelles d'une cinquantaine de films.

Le sujet est tellement vaste qu'il a fallu organiser les films de la Spatiosphère autour de sept thématiques dans lesquelles les établissements culturels se reconnaissent, et qui permettent aux visiteurs d'approfondir les centres d'intérêts qui leur sont propres. Le thème "Univers » regroupe des films sur l'étude des étoiles, des planètes et du cosmos en général. Le thème " Terre ", ceux sur la machine Terre, la Terre géophysique avec ses éventuelles composantes anthropiques. Le thème «Vie » couvre tous 


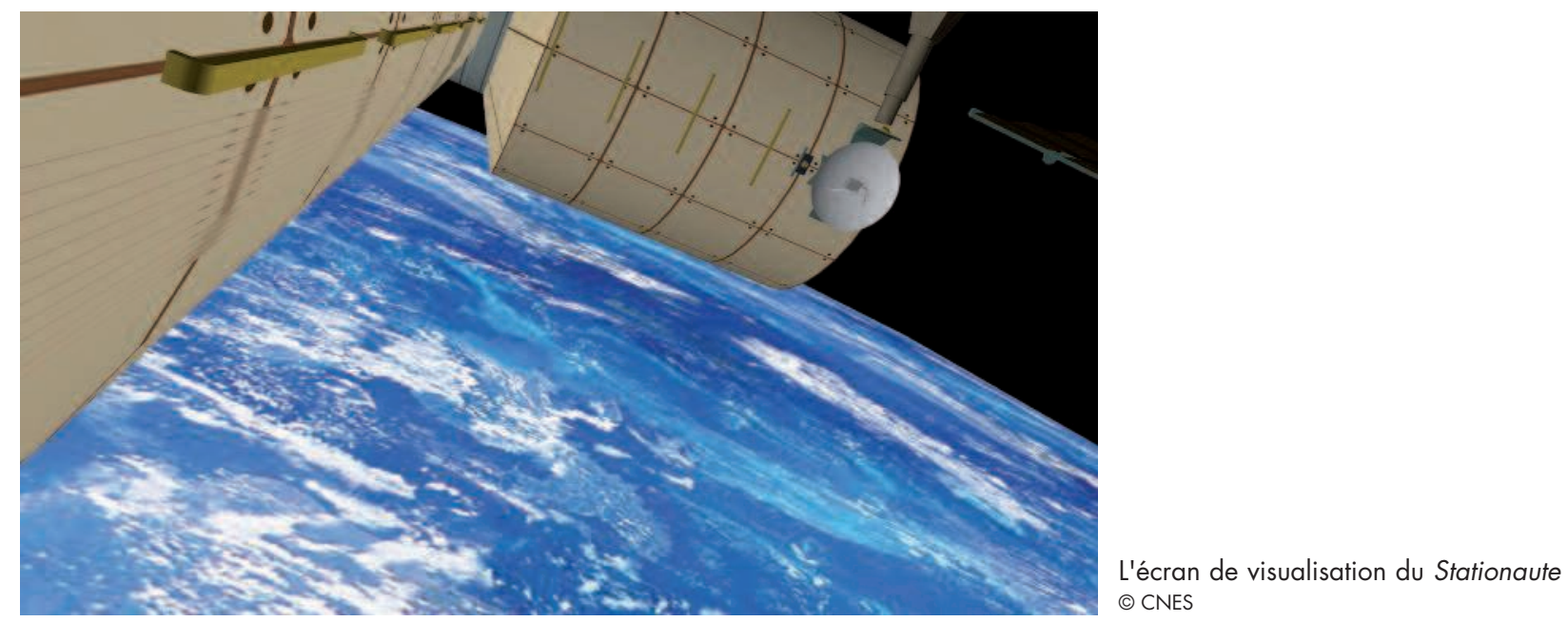

Stationaute trouve un usage surprenant pour traduire les métaphores du mouvement d'un corps dans l'Espace.

\section{L’impact dans les musées}

Il était important d'apprécier si le but, qui était d'offrir un autre mode d'accès aux apports de l'Espace, a pu être atteint par les dispositifs interactifs qui sont actuellement déployés dans des établissements culturels. Nous sommes conscients que seule une enquête de public pourrait satisfaire à cet objectif. Dans un premier temps, nous avons seulement recueilli quelques impressions auprès de ceux de leurs responsables qui les utilisent, ou qui les ont simplement expérimentés en démonstration.

L'Hydronaute est le dispositif actuellement le plus déployé dans les musées. Yves Le Maner, directeur de la Coupole, centre d'Histoire et de Mémoire à Saint-Omer, trouve que l'Hydronaute est une remarquable clef d'entrée à la compréhension de la Terre vue de l'Espace, et de sensibilisation à sa complexité. De son avis, il gagne bien sûr en intérêt lorsqu'il est mis en perspective par un pédagogue formé à son maniement, ce qui n'est pas toujours le cas.

Eric Hussenot, directeur d'Océanopolis, qui a installé l'Hydronaute à Brest énonce qu'il est un élément central, le plus utilisé dans l'espace océanographique qui comporte pourtant neuf bornes. Il a choisi de projeter l'image sur un écran de deux mètres de base, dans une salle de $150 \mathrm{~m}^{2}$, qui permet une animation pédagogique, soit par le professeur qui y emmène sa classe, soit par un animateur du musée.
Jean-Paul Dekiss, directeur du centre international Jules Verne à Amiens, a mis l'Hydronaute en scène dans un module qui ressemble à un très grand LEM posé sur la Lune, dans le noir, connecté à un grand écran car il pense que c'était un outil précieux pour tous les publics. Il lui a été très utile dans son exposition qui comparait la vision futuriste, terrestre ou sous-marine, de Jules Verne, et ce qui est le point actuel de nos connaissances.

Tous effectivement, constatant la complexité du contenu, soulignent l'apport d'un pédagogue qui accompagne les démonstrations.

Marc Moutin directeur des programmes, commente avec enthousiasme le Stationaute qui est déployé à la Cité de l'Espace. "L'utilisateur vit en trois dimensions la journée d'un spationaute. Il se déplace avec lui, dans ce milieu inimaginable de l'impesanteur, observe les diverses expériences scientifiques qu'il effectue durant son séjour en orbite, qui toutes auront une incidence sur des aspects, connus ou à venir, de la vie terrestre. Les explorations de l'utilisateur s'entrecroisent, il arrive tout d'un coup à un endroit où il a quelque chose à faire, placer sur lui une électrode, se prêter à une échographie en impesanteur... J'ai couplé le Stationaute à un écran carré de deux mètres. Le palet permet de se déplacer dans trois directions, de communiquer à son corps une accélération vers l'avant ou l'arrière, faire demi-tour dans l'espace, glisser le long d'une paroi de la station... Je ne dispose pas encore de la Wiimotte. Elle donne certainement un grand sentiment de liberté, mais pose encore quelques problèmes de sécurité et d'ergonomie. Dans notre salle "Vivre dans l'Espace", le Stationaute est entouré d'autres moyens d'approche, mais c'est lui qui a le plus grand succès ». 
L'Orbiscope, qui n'est pas encore déployé, a pu néanmoins être essayé par Yannick Thevenin, directeur du musée des Transmissions près de Rennes, Marc Moutin et Yves Le Maner. Ce dernier résume le sentiment commun avec le constat que ce dispositif pourrait s'intégrer avec profit dans une mise en perspective historique proposée aux visiteurs.

La Spatiosphère qui est actuellement présentée dans deux muséums pose moins de questions quant à son ergonomie et tant Gérard Ferrière, directeur du muséum de Dijon, qu'Armand Fayard, directeur du muséum de Grenoble, estiment que la Spatiosphère apporte des développements éclairants sur les points de vue développés dans leurs expositions.

\section{Le choix d'une politique publique}

Chacun de ces outils est conçu comme une expérience unique que tout visiteur peut accomplir lors de sa visite au musée. Il va de soi que l'investissement considérable que représentent la conception et la réalisation de dispositifs d'une telle richesse de contenu est totalement pris en charge par l'Observatoire de l'Espace du CNES. Seul un grand organisme peut assumer le coût de la création et de la mise à jour des logiciels qui les organisent et les gèrent. Pour ce faire, il s'appuie sur les compétences d'un industriel, partenaire depuis le début du projet : C.S. Communication et Système qui s'est investi pleinement dans ces recherches (logiciels, ergonomie, traitement des données, nouveaux périphériques). C'est lui qui installe les applications dans les musées, assure la maintenance, et demeure leur interlocuteur technique. Le fait de s'appuyer sur une importante structure industrielle a permis d'année en année la construction d'une famille de dispositifs interactifs aussi innovante. Mais c'est la position centrale de l'Observatoire de l'Espace au carrefour de la communauté scientifique, des acteurs du spatial et des industriels qui a facilité la réunion des compétences, des informations, et des données indispensables à leur conception. Il peut ainsi piloter la fabrication d'outils aussi spécialisés, par définition non rentables financièrement, mais culturellement indispensables. Il remplit là une mission de service public, au sens le plus légitime et le plus justifié du terme. C'est le choix politique de départ : l'Observatoire de l'Espace du CNES a été créé pour que chacun prenne l'indispensable conscience de l'intégration de l'Espace dans la culture quotidienne. Participer à cette mission entre pleinement en résonance avec les missions des établissements culturels concernés - et tous le sont. Le coût individuel de ces dispositifs représente pour eux une part très réduite de l'énorme investissement de départ. Cette coopération financière est indispensable : elle marque leur détermination à participer à cette aventure, à la promouvoir, et élargit la mission que chacun assume. Mais elle ne doit pas grever leur budget au point qu'ils renoncent à coopérer à la diffusion de connaissances présentées sous un aspect ludique mais qui, dans leur exhaustivité, favorisent la réflexion. Quand on prend la mesure de l'importance de l'aventure spatiale pour la gestion de ses ressources, la prévision des changements climatiques bénéfiques ou néfastes, le devenir de l'humanité, il semble nécessaire de mettre ces outils de savoir et de culture scientifique à la disposition du plus grand nombre.

Dans l'avenir, notre projet est de continuer d'investir dans de nouveaux moyens qui renouvellent les représentations de notre monde, qui enrichissent les connaissances de chacun et leurs ouvrent des perspectives sur de nouvelles expérimentations. Cette détermination repose sur la confiance que nous plaçons dans nos partenaires culturels pour participer et relayer cette œuvre de science et d'imagination. 